\title{
Three-Dimensional Laser Imaging as a Valuable Tool for Specifying Changes in Breast Shape After Augmentation Mammaplasty
}

\author{
Danielle L. Esme · Alexander Bucksch • \\ Werner H. Beekman
}

Received: 9 May 2008/Accepted: 23 September 2008/Published online: 4 November 2008

(c) Springer Science+Business Media, LLC and International Society of Aesthetic Plastic Surgery 2008

\begin{abstract}
Background Three-dimensional (3D) terrestrial laser scanning (TLS) is a valuable method for measuring shapes of objects and for obtaining quantitative measurements. These qualities of the 3D laser scanner have proved to be useful in reconstructive breast surgery. This study investigated various 3D parameters to obtain an optimal objective visualization of the breast after cosmetic augmentation mammaplasty.

Methods The objects are represented in a point cloud, which comprises millions of $x, y$, and $z$ coordinates representing a virtual image. The quantification of 3D points shows changes in height ( $z$ coordinate) at any chosen point on the augmented breast ( $x$ and $y$ coordinates). To give visual feedback on the change in dimensions, a color elevation scheme was applied on the reconstructed surface of the breast. As a quantifying description, a sagittal B-spline was chosen in a plane through the nipple to obtain the breast shape via the lateral profile.

Results Pre- and postoperative clear images were obtained. The color elevation model showed an increased
\end{abstract}

D. L. Esme $(\bowtie) \cdot$ W. H. Beekman

Department of Plastic and Reconstructive Surgery,

Tergooiziekenhuizen Hilversum, Van Riebeeckweg 212,

1213 XZ Hilversum, The Netherlands

e-mail: danielle@sandan.nl

\section{A. Bucksch}

Faculty of Aerospace Engineering, Delft Institute of Earth Observation and Space Systems (DEOS), Delft University of Technology, Delft, The Netherlands projection and upper pole fullness after augmentation. The B-spline showed the gain in projection in a sagittal plane through the nipple.

Conclusions Three-dimensional TLS is capable of objectifying changes in shape after augmentation mammaplasty. This imaging technique represents superior visualization of the breast shape and can serve as a valuable tool to determine the changing dimensions of the breasts after augmentation mammaplasty.

Keywords 3D Terrestrial laser scanning . Augmentation mammaplasty

In recent years, terrestrial laser scanning (TLS) has become a standard tool in three-dimensional (3D) shape measuring for a wide variety of objects including construction works, industrial plants, forestry, and buildings [1, 3, 10, 23]. With TLS, it is possible to measure millions of $3 \mathrm{D}$ points in a few seconds and to create highly detailed and accurate computer models of a shape. It is a rapidly developing application for obtaining the shape, contour, size, and volume of objects.

The medical field also may benefit from advances in 3D imaging [5, 8, 12, 21]. In plastic surgery, 3D modeling offers unique opportunities in reconstructive breast surgery [9], providing visual and quantitative assessment of reconstructed breasts $[9,11]$. Until recently, no techniques were available to objectify the change in the breasts after cosmetic breast implant surgery.

In our study, we performed intraoperative 3D laser imaging before and directly after implantation to assess the contour, shape, and volume changes of the breast after implantation of silicone gel-filled breast prostheses. 


\section{Materials and Methods}

Patient

A 31-year-old healthy woman who presented at our department for an augmentation mammaplasty was randomly selected for our study. She had no history of breast surgery. The patient presented with a cup size of 75AA, a body weight of $62 \mathrm{~kg}$, and a height of $169 \mathrm{~cm}$.

Methods

\section{Measurement Setup}

In our setup, a FARO HS 880 TLS (Eindhoven, the Netherlands) was used. The scanner is a phase-based system that measures the distance from the scanner to an object surface at a near infrared (NIR) wavelength of $782 \mathrm{~nm}$ (Fig. 1). A phase-based laser scanner relies on a bior triphase modulation of the laser light frequency. For the distance measurement, the phase difference of the modulated wave is taken to calculate the distance between the object and the scanner.

The measurement was performed with a continuous laser beam, which has a continuously changing phase angle to achieve high scanning speeds. The scanning speed is approximately 125,000 points per second. By minimizing the scan angle to $20^{\circ}$, the scanning time was reduced to $20 \mathrm{~s}$ [4]. To edit the data, the Cyclone 5.4 software package (Leica Geosystems, Heerbrugg, Switzerland) was used.

\section{Perioperative Procedures}

Key anatomic landmarks on the breast were chosen and used as reference points [2, 15, 27]. The scanner was positioned in front of the patient. An adjustable tripod enabled us to get a $90^{\circ}$ angle between the longitudinal thoracic axis of the patient in a $45^{\circ}$ supine position and the optical center of the laser scanner (Fig. 2).

After general anesthesia, the first series of thoracic scans were made with the patient in the $45^{\circ}$ supine position. Once the patient was positioned horizontally, a dual-plane pocket

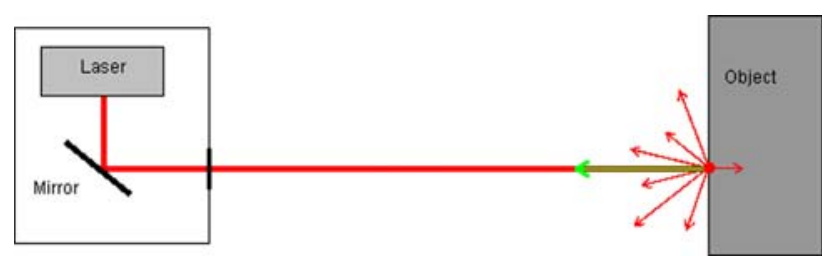

Fig. 1 Measuring principle of a terrestrial laser scanner. Left: The mirror inside the laser scanner that deflects the laser signal in a certain direction. Right: The measured object on which the signal is reflected. The green arrow is the echo returning to the laser scanner

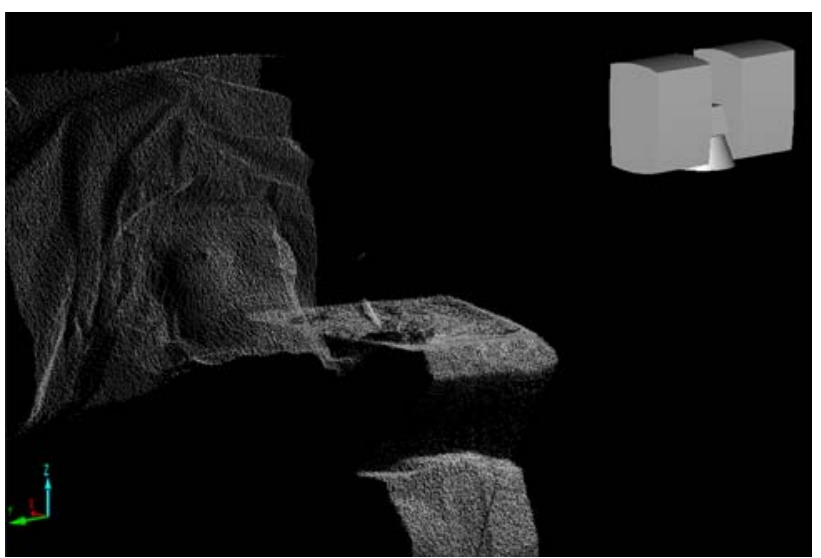

Fig. 2 Setting in the operating room with the scanner positioned in front of the patient. The patient is in a $45^{\circ}$ supine position

[25] was created through an inframammary incision. After performance of the augmentation using a style 410 prosthesis with a volume of $290 \mathrm{~g}$ (McGhan Style 410; Inamed Aesthetics, Santa Barbara, CA, USA), the patient again was positioned at the $45^{\circ}$ angle, and a second series of scans was performed.

\section{Data Processing}

In both recordings, the resulting points were in a spherical coordinate system around the optical center of the laser scanner. By using two reference points and a reference plane (the surgery table), the two scans were placed into the same Cartesian coordinate system $x$-axis (left/right), $y$ axis (superior/inferior), and $z$-axis (anteroposterior). This allowed us to compare the two scans. To quantify the gain or loss of distance in the millions of 3D points, we extracted the 50 points that were distributed over the thoracic wall. We compared the changes in height ( $z$ coordinate) at any chosen point $(x, y$ coordinate) of the augmented breast with the preoperative breast.

As a visual feedback, an elevation model of both scans was created. This elevation model was made using all points and provided changes in height at a certain position of the breast represented by a color scheme. Each color represented a certain gain or loss in a posteroanterior projection. As a second analysis, we used a sagittal profile at the level of the nipple, a B-spline [22], which was constructed from the point cloud.

\section{Results}

The 3D processing of the scanned patient before and after augmentation presented clear images (Fig. 3). The quantification of the 3D points shows changes in height ( $z$ coordinate) at any chosen point on the augmented breast 

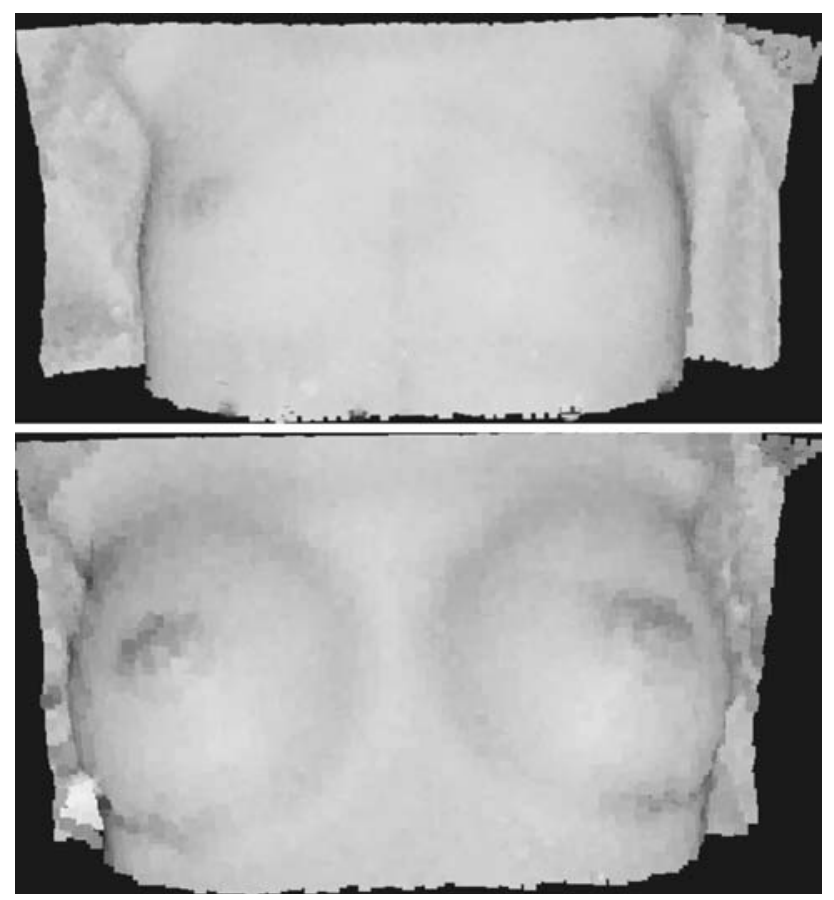

Fig. 3 Three-dimensional imaging before (top) and after (bottom) augmentation with a 290-g biodimensional prostheses (McGhan Style $410 \mathrm{MX})$

( $x, y$ coordinate). With a maximum prosthesis projection of $5.5 \mathrm{~cm}$, the maximum gain in the projection of the breast is $4.8 \mathrm{~cm}$. The color scheme elevation model shows the objective visual feedback with the concomitant changes in dimension (Fig. 4). An almost flattened appearance before augmentation is seen. An increased projection, with a concentric circular pattern around the areola and upper pole fullness, is seen postoperatively. The B-spline shows the sagittal view of the gain in projection through the nipple (Fig. 5).

\section{Discussion}

Cosmetic breast augmentation using silicone gel-filled breast prostheses currently is a commonly performed plastic surgery procedure [24]. For the past three decades, approximately 1 million U.S. women have received silicone breast implants [26]. In performing augmentation mammaplasty, plastic surgeons need objective tools to measure the postoperative dimensional changes of the breasts.

In a previous study, we showed, with the use of a 3D magnetic resonance imaging (MRI) technique, that the in vitro 3D configuration of the round and anatomically shaped silicone gel-filled breast prostheses will hardly change in vivo after subpectoral augmentation [20]. This offers opportunities for preoperative determination of what
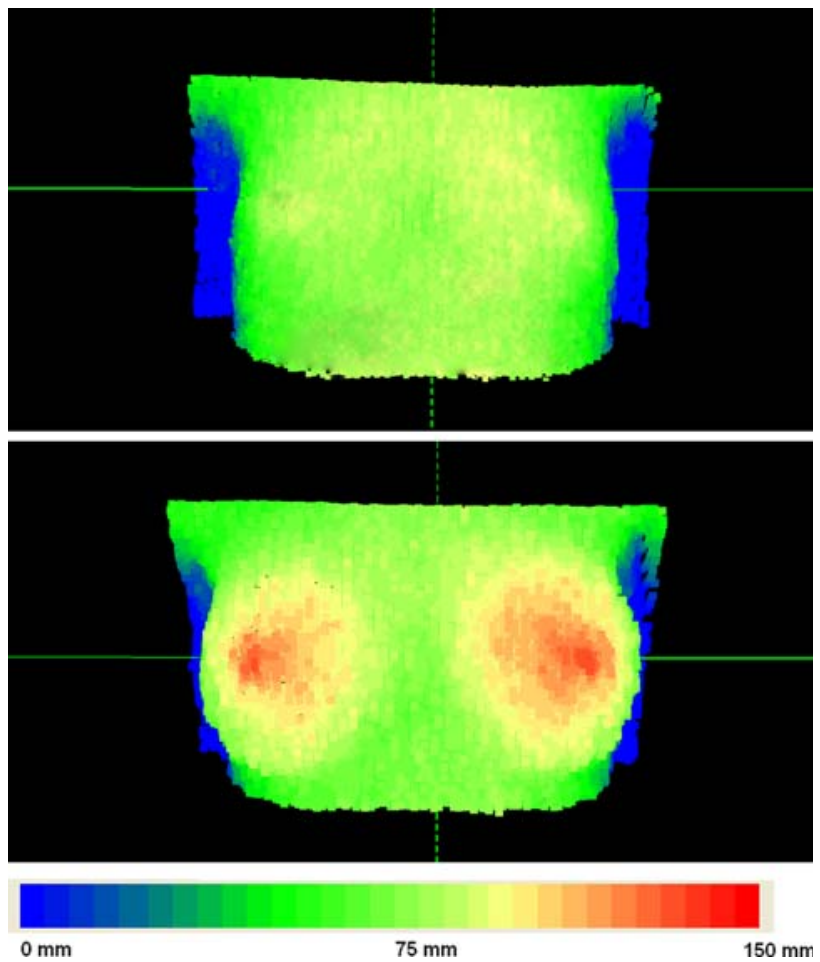

Fig. 4 Color scheme elevation model before (top) and after (bottom) augmentation with a 290-g biodimensional prostheses (McGhan Style $410 \mathrm{MX}$ )

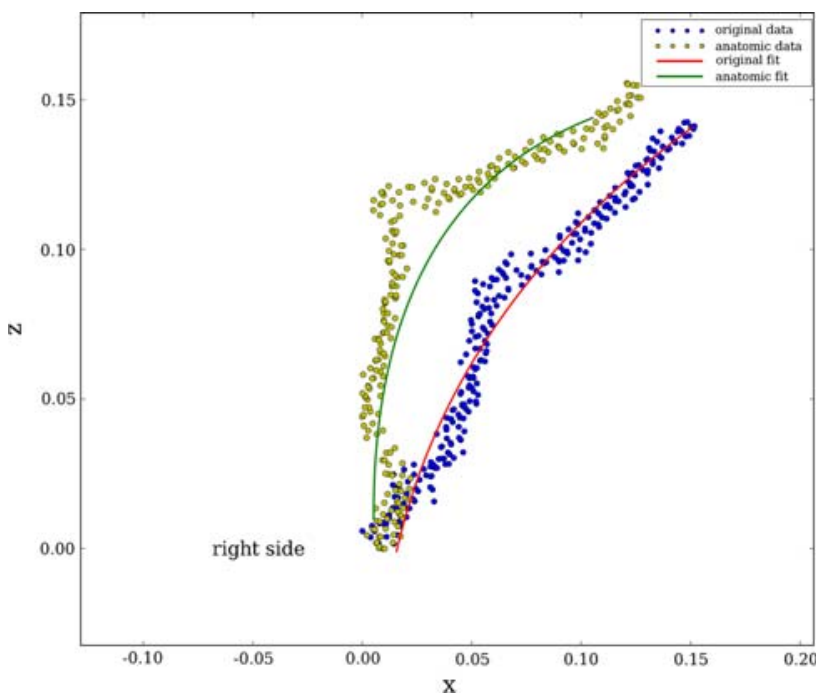

Fig. 5 The B-spline showing the sagittal view of the gain in projection through the nipple before and after augmentation with a 290-g biodimensional prostheses (McGhan Style 410 MX)

prosthesis shape to use in a certain type of breast. However, even if a certain type of prosthesis holds its form after implantation, the question remains what it will contribute to the shape of the breast. To date, no study has been performed to objectify the change in outer shape of the breast after cosmetic breast augmentation mammaplasty. 
Recently, only standard 2D images were available for obtaining the postoperative shape, offering no possibilities for calculating the exact change in dimensions at any given point of the augmented breast. In general, 3D analysis offers several advantages over other methods of measuring breast shape and volume $[6,7,9,13,15,16-19]$.

As a new technology, TLS has several advantages over other laser scanners. The TLS, a single handheld portable device, is insensitive to light conditions and skin colors, is not invasive because of the low-output laser light [11], has a quick measurement time, and is highly accurate.

Only one recording step is needed to capture the breast profile and volume. Subsequently, only a short time is needed to generate a 3D point cloud offering a perfect image of the augmented breasts. With the presented 3D representation, we are able not only to visually assess the size, shape, and contour of the breast, but also to obtain the quantitative shape and contour change of the breast.

Isogai et al. [11] quantitatively analyzed the reconstructed breast with a 3D laser light scanner. Using the Moire pattern analysis, they constructed topographic surface images of the breast. Our color scheme elevation model, however, shows not only the objective visual feedback, but also the concomitant changes in dimension reproducing data ( $x, y$, and $z$ positions) at each point on the surface of the breast.

The noncontact approach was ideal for intraoperative use. No adjustments or compromises to the standard surgery environment were necessary. In an outpatient department setting, it is highly important to ensure exact scanning positions of the patient both pre- and postoperatively. Kovacs et al. [14] used dummy models to optimize the 3D imaging of the breast region with a 3D laser scanner. Our setting was chosen to minimize the changes in the position of the patient and the TLS and thereby to optimize the precision of the measurements.

Although the intraoperative laser scanning was easily performed, we are aware that our study still has its limitations. Our goal is to optimize the outpatient department conditions to be certain of reproducible imaging both preand postoperatively. In scanning 6 weeks postoperatively, the effects of the direct postoperative changes such as skin tension and swelling are reduced to objectify the final results ideally.

\section{Conclusion}

In our opinion, 3D TLS can be of great importance in objectifying breast augmentation surgery results. With the 3D coordinate system, it is possible to obtain the postoperative change of dimension at any point on the breast after augmentation. This will enhance postoperative surgery documentation and may contribute to future preoperative planning.

\section{References}

1. Becker S, Haala N (2007) Combined feature extraction for facade reconstruction. ISPRS workshop on Laser Scanning and SilviLaser 2007, Espoo, Finland

2. Brown TP, Ringrose C, Hyland RE, Cole AA, Brotherston TM (1999) A method of assessing female breast morphometry and its clinical application. Br J Plast Surg 52:355-359

3. Bucksch A, Lindenbergh R (2007) A skeletonization method for point cloud processing ISPRS. J Photogramm Remot Sens (submitted)

4. Bucksch A, Lindenbergh R, Ree J (2007) Influence of the intensity remission on the scan quality. In: Presented at the symposium on the error budget of terrestrial laserscanning, GeoSiberia, Novosibirsk, Russia

5. Bush K, Antonyshyn O (1996) Three-dimensional facial anthropometry using a laser surface scanner: validation of the technique. Plast Reconstr Surg 98:226-235

6. Edsander-Nord A, Wickman M, Jurell G (1996) Measurement of breast volume with thermoplastic casts. Scand J Plast Reconstr Surg Hand Surg 30:129-132

7. Farkas LG, Bryson W, Klotz J (1980) Is photogrammetry of the face reliable? Plast Reconstr Surg 66:346-355

8. Ferrario VF, Sforza C, Poggio CE, Tartaglia G (1994) Distance from symmetry: a three-dimensional evaluation of facial asymmetry. J Oral Maxillofac Surg 52:1126-1132

9. Galdino GM, Nahabedian M, Chiaramonte M, Geng JZ, Klatsky S, Manson P (2002) Clinical applications of three-dimensional photography in breast surgery. Plast Reconstr Surg 110:58-70

10. Gorte B (2007) Skeletonization of laser-scanned trees in the 3D Raster domain. Presented on a symposium, 3DGeoInfo2006, Malaysia

11. Isogai N, Sai K, Kamiishi H, Watatani M, Inui H, Shiozaki $H$ (2006) Quantitative analysis of the reconstructed breast using a 3-dimensional laser light scanner. Ann Plast Surg 56:237-242

12. Kaneko T (1993) A system for three-dimensional shape measurement and its application in microtia ear reconstruction. Keio J Med 42:22-40

13. Kouchi M, Mochimaru M, Tsuzuki K, Yokoi T (1996) Random errors in anthropometry. J Hum Ergol (Tokyo) 25:155-166

14. Kovacs L, Zimmermann A, Papadopulos NA, Biemer E (2004) Factors determining shape and symmetry in immediate breast reconstruction. Ann Plast Surg 53:192-194

15. Kovacs L, Yassouridis A, Zimmermann A, Brockmann G, Wohnl A, Blaschke M, Eder M, Schwenzer-Zimmerer K, Rosenberg R, Papadopulos NA, Biemer E (2006) Optimization of 3-dimensional imaging of the breast region with 3-dimensional laser scanners. Ann Plast Surg 56:229-236

16. LePage MA, Kazerooni EA, Helvie MA, Wilkins EG (1999) Breast reconstruction with TRAM flaps: normal and abnormal appearances at CT. Radiographics 19:1593-1603

17. Loughry CW, Sheffer DB, Price TE Jr, Lackney MJ, Bartfai RG, Morek WM (1987) Breast volume measurement of 248 women using biostereometric analysis. Plast Reconstr Surg 80:553-558

18. Loughry CW, Sheffer DB, Price TE, Einsporn RL, Bartfai RG, Morek WM, Meli NM (1989) Breast volume measurement of 598 women using biostereometric analysis. Ann Plast Surg 22:380-385

19. Mineyev M, Kramer D, Kaufman L, Carlson J, Frankel S (1995) Measurement of breast implant volume with magnetic resonance imaging. Ann Plast Surg 34:348-351

20. Nipshagen MD, Beekman WH, Esme DL, de Becker J (2007) Anatomically shaped breast prosthesis in vivo: a change of dimension? Aesth Plast Surg 31(5):540-543 
21. O'Grady KF, Antonyshyn OM (1999) Facial asymmetry: threedimensional analysis using laser surface scanning. Plast Reconstr Surg 104:928-937

22. Prautzsch H, Boehm W, Paluszny M (2002) Bezier and B-spline techniques. Mathematics and visualization, XIV. ISBN 978-3-54043761-1

23. Rabbani T (2007) Automatic reconstruction of industrial installations using point clouds and images. $\mathrm{PhD}$ thesis, Delft University of Technology

24. Rohrich RJ (2000) The increasing popularity of cosmetic surgery procedures: a look at statistics in plastic surgery. Plast Reconstr Surg 106:1363-1365
25. Tebbetts JB (2006) Dual-plane breast augmentation: optimizing implant-soft tissue relationships in a wide range of breast types. Plast Reconstr Surg 118:81S-98S

26. Van Zele D, Heymans O (2004) Breast implants: a review. Acta Chir Belg 104:158-165

27. Westreich M (1997) Anthropomorphic breast measurement: protocol and results in 50 women with aesthetically perfect breasts and clinical application. Plast Reconstr Surg 100:468-479 